\title{
Expertos, peritos y actuación pericial: nuevos horizontes profesionales en torno al libro antiguo y raro
}

\author{
Por Manuel-José Pedraza-Gracia
}

\begin{abstract}
Resumen: Se analizan las actividades de valoración y tasación de libros antiguos y raros desde el ejercicio profesional como perito o experto en estos documentos. Se parte de la necesidad de expertos en estos documentos para la asesoría e intermediación entre los participantes en negocios en los que intervienen documentos antiguos y raros y sobre los problemas que se presentan durante las actividades que se realizan con ellos. Se proporciona una aproximación a la condición de perito, se perfila el ámbito de actuación y sus orígenes y causas, se ofrece una tipología de actuaciones y se establece un procedimiento de actuación. Se pone de manifiesto la capacidad de los documentalistas para realizar estas actividades.

Palabras clave: Libro antiguo, Libro raro, Perito, Tasación, Valoración, Expertización, Peritación.

Title: Expert and technical activities: old and rare books offer new professional horizons

Abstract: The activities of valuation and appraisal of old and rare books are analyzed as a professional service to be provided by experts in the field. First, a general outline is presented on the need of such experts as advisers and intermediaries between the participants in the business of acquiring and selling old and rare documents. An approximation to the status of such an expert is provided, including scope of action, origins and causes, and detailing a typology of the most frequent tasks in which he/she may be involved and a comprehensive procedure for this professional course of activity.
\end{abstract}

Keywords: Old books, Rare books, Expert, Valuation, Appraisal, Expertization, Expert opinion.

Pedraza-Gracia, Manuel-José. "Expertos, peritos y actuación pericial: nuevos horizontes

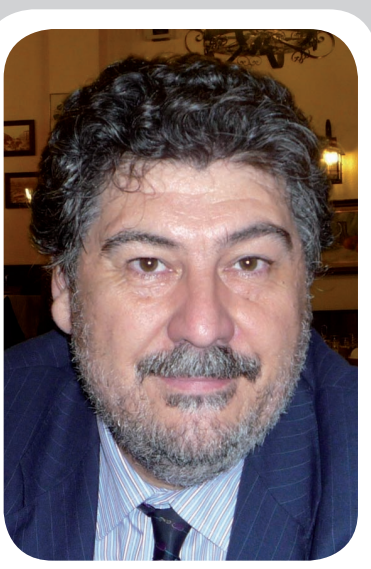

Manuel-José Pedraza-Gracia es bibliotecario y profesor titular del Área de Biblioteconomía y Documentación en la Universidad de Zaragoza. Ha trabajado en temas relacionados con la bibliografía, la historia del libro, el libro antiguo y la tasación de documentos antiguos. Dirige los cursos sobre tasación y valoración de libro antiguo de la Universidad de Zaragoza en Jaca. profesionales en torno al libro antiguo y raro". El profesional de la información, 2010, mayo-junio, v. 19, n. 3, pp. 269276.

DOI: 10.3145/epi.2010.may.07

\section{Introducción}

El documento antiguo y el libro de valor se han convertido en uno de los problemas de gestión documental más importantes entre los propietarios, administradores y gestores de bibliotecas, en particular, y del patrimonio en general.

Desde una perspectiva eminentemente política, el libro antiguo o una primera edición de un autor de la generación del veintisiete o un raro contemporáneo resultan ser hoy, sobre todo, generadores de problemas ${ }^{1}$, a la par que conforman la parte más apreciada de un patrimonio bibliográfico que es preciso controlar y que causa gastos que no generan el rédito político y de gestión que los justifique.

Desde el punto de vista documental es complejo a la hora de identificar y tratar, es costoso de preservar, es difícil de conservar, tiene un público reducido pero experto conocedor del material que estudia, frecuen- temente hay que saber latín (o griego) para leerlo (y tratarlo) y ocupa mucho espacio. El gestor de estos documentos suele encontrar desproporcionada la relación entre su precio y el coste de mantenimiento, por una parte, y el uso que se hace de él, por otra. El propietario de estos documentos desconfía de los investigadores y de la legislación sobre patrimonio bibliográfico por interferir en su propiedad. El librero anticuario considera que su libertad de comercio está restringida por la normativa.

Pero, pese a su condición de objeto problemático, la puesta en valor de estos documentos ha llegado a unos límites en los que la relación valores-inconvenientes (incluyendo entre los valores el económico) hace que el interés por el libro antiguo se esté incrementando desde todas las perspectivas que se han mencionado previamente. Este interés se ve respaldado por la existencia de especialistas que reconocen los valores que estos documentos poseen para la sociedad, para la 
institución o el particular que los posee y para el usuario que los consulta e investiga.

\section{"El libro raro de calidad (antiguo, o no) es un activo seguro"}

Si el análisis de los valores se reduce de forma exclusiva al económico, el libro raro de calidad (antiguo, o no) es un activo seguro (Flores-Jimeno, 2005), un producto de comercio internacional muy rentable, aunque escaso. Este documento puede surgir en cualquier lugar del mundo y en condiciones diversas ${ }^{2}$ y se puede poner en circulación por diferentes medios: el mercado anticuario tradicional, la subasta ${ }^{3}$, en la red o en el mercado irregular. En este mundo del comercio de documentos, el conocimiento de lo que se posee o gestiona, de sus valores y precio, resulta esencial tanto para su gestión como documento, como para el fondo existente en un centro, o para el centro que lo posee, en cuanto organización.

Hay muchos avatares que interfieren en los valores de un documento, incluido el crematístico, y cada vez más se producen situaciones en las que se precisa establecer la autenticidad del mismo en todo o en parte. No es infrecuente que lo que se pretende sea determinar la pérdida de valor producida en un documento por cualquier causa o accidente de cara a exigir las responsabilidades pertinentes. Otras muchas actuaciones exigen precisar un precio acorde a los valores que posee el documento para su propietario, generalmente relacionados con un seguro (precio que no tiene por qué ser el mismo en todos los casos), con préstamos para una exposición, con la salida del documento para una restauración o digitalización, con donaciones, con depósitos, con un robo, con un accidente...

En ocasiones (y cada vez va a ser más frecuente), es necesario conocer el valor económico de una biblioteca a efectos de concretar el patrimonio real de la institución propietaria de la misma, o de una compraventa, de una donación, de una herencia... Pero también se necesita averiguar con la mayor exactitud posible los valores de los documentos. Esto exige una profundización en su comprensión que no todos los ciudadanos y aun los propios profesionales de la documentación poseen. Por esta causa, el particular que se interesa por una propiedad cuyo alcance y autenticidad desconoce, pero intuye, se encuentra en la necesidad de ser asesorado por un experto. Además, el peso del valor económico de estos materiales favorece una más alta incidencia de problemas y discrepancias en la multitud de actuacio- nes que se pueden llevar a cabo en las que intervienen llegando, en ocasiones, al litigio. Algunas de estas discrepancias requieren intermediarios que puedan ayudar a alcanzar un acuerdo o que asesoren en su resolución $y$, en el caso de acabar en litigio, la presencia y actuaciones de peritos están perfectamente reguladas en la legislación procesal.

Por último, el centro que posee las obras requiere que, entre las herramientas para su gestión, esté perfectamente definido su conocimiento, lo más profundo posible, tanto de su precio como del resto de sus valores.

\section{"La presencia y actuaciones de peritos están reguladas en la legislación procesal"}

\section{Experto y perito}

La definición de experto tiene su origen en la palabra experiencia: "tener la práctica prolongada que proporciona conocimiento o habilidad para hacer algo" (segunda acepción en el Diccionario de la Real Academia Española). La Academia identifica como sinónimos los términos experto y perito, que es "el entendido, experimentado, hábil, práctico en una ciencia o arte".

Esta misma autoridad en su tercera acepción, relacionando el término con el derecho, indica que perito es la "persona que, poseyendo determinados conocimientos científicos, artísticos, técnicos o prácticos, informa, bajo juramento, al juzgador sobre puntos litigiosos en cuanto se relacionan con su especial saber o experiencia". Si algo queda claro de estas definiciones es que cualquier actuación de las mencionadas anteriormente precisa conocimiento y experiencia, sinónimo, a su vez, de pericia: "sabiduría, práctica, experiencia y habilidad en una ciencia o arte", en el caso que se trata, en el libro antiguo, raro o de valor contrastado.

La propia legislación a la hora de establecer quién posee la condición de perito define dos vías: o bien la posesión de un título oficial en una ciencia o arte cuyo ejercicio esté regulado por la administración, o bien tener conocimientos o prácticas especiales en alguna ciencia o arte, aun careciendo de título oficial ${ }^{4}$. Por tanto, cualquier persona, incluso jurídica, puede ser perito con tal de que tenga experiencia y, en principio, el perito puede aceptar o rechazar el encargo.

Entre los derechos del perito está la percepción de honorarios y, entre los deberes, la elaboración del dictamen, conforme a las reglas de su profesión, mediante una actuación lo más objetiva posible, lo que se debe 
manifestar expresamente bajo juramento o promesa de decir la verdad. También es su obligación no distorsionar, omitir o faltar a la verdad falseando el informe, lo que puede dar lugar a sanciones penales. El perito debe asistir al juicio o la vista donde se practicará la prueba.

\section{Actuación pericial}

Cualquier actuación pericial aporta siempre y únicamente un juicio de valor de un profesional sobre un asunto específico (justamente lo que se pretende) dotado de plena objetividad ${ }^{5}$ frente al objeto y frente al interesado o a los interesados. Pero en determinadas cuestiones puede ser contrastado necesariamente con otros. De hecho, en el proceso criminal se exige que el reconocimiento pericial sea realizado de forma obligatoria por dos peritos ${ }^{6}$.

\section{"Cualquier persona, incluso jurídica, puede ser perito con tal de que tenga experiencia"}

Casi todas estas actuaciones tienen como resultado un informe técnico o dictamen, generalmente elaborado por escrito, que comprende diversos datos, informaciones y consideraciones que se pueden emplear consecuentemente al uso que se le pretenda dar o a la solicitud del mismo. De esta manera, tras una pericia se obtiene, casi siempre, un documento técnico avalado por un experto que lo firma y que garantiza la información que el documento contiene. En algunas ocasiones esa afirmación se puede hacer más relevante cuando el documento no sólo la garantiza, sino que la certifica. Interviene, por consiguiente, además del componente de experiencia, un componente ético por parte del experto y, al mismo tiempo, de confianza en el perito por parte de los comitentes que son quienes encargan su actuación. Este componente ético es esencial en la pericia y difícilmente puede ser considerado como perito o experto válido el profesional que posteriormente pueda verse beneficiado por su decisión, bien sea por participar en el negocio, por tener cualquier tipo de relación con el mismo o porque su decisión pueda influir en negocios de carácter similar en los que esté o pueda estar implicado ${ }^{7}$.

Este tipo de actuaciones requiere primero la consulta del documento original (siempre que sea posible) y la comparación con otros elementos o unidades similares para establecer las características definitorias que se reseñan en el informe sobre las actuaciones, tanto más, cuanto menos normas existan para establecer y fundamentar la opinión. Si bien este tipo de comparaciones pueden realizarse en exclusiva con respecto a los conocimientos y experiencias prácticos del responsable del informe o dictamen.

\section{"La expertización es la actuación pericial por la que el experto efectúa una detallada relación descriptiva de todos los elementos del objeto examinado"}

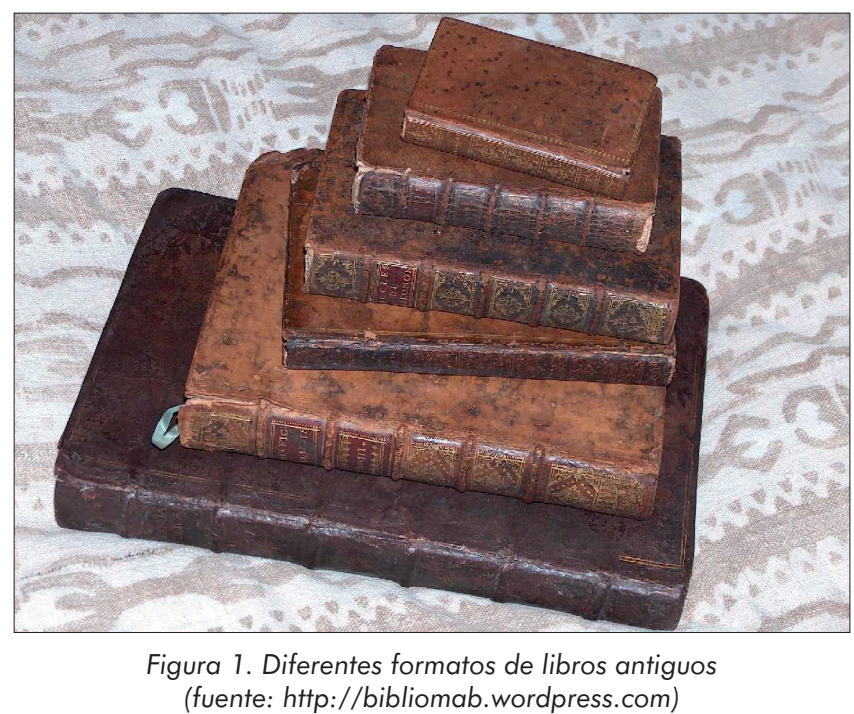

\section{Tipos de actuaciones periciales}

Si se entra en la casuística de estas actuaciones, se aprecia que resulta suficientemente variada como para poder diferenciar tipos de actos periciales tomando como elemento clasificador determinadas variaciones y particularidades (Liberati, 2005, pp. 213-222). Pueden diferenciarse perfectamente, siendo el elemento distintivo la finalidad o uso que se pretenda dar a la pericia, las siguientes actuaciones:

a) Expertización: es la actuación pericial por la que el experto efectúa una detallada relación descriptiva de todos los elementos del objeto examinado. Contiene el parecer motivado del experto y se centra en la identificación y en la autentificación de la unidad analizada. Suele solicitarse para establecer la autenticidad de una obra, su estado de conservación, su identificación, etc. Es, por tanto, la actuación que conduce al establecimiento de los valores del libro. La expertización puede y debe entenderse como sinónimo de investigación y a todos lo efectos produce una certificación sustentada en los conocimientos del experto. Éste deberá individualizar el objeto de estudio de forma inequívoca para evitar que se produzca cualquier fraude posterior utilizando 
copias del elemento estudiado validadas por su certificación, por lo que debería incorporar las reproducciones fotográficas necesarias para que acompañando al objeto en cuestión no deje duda de que se refiere a él.

En el caso del libro antiguo se expertiza la identificación de la edición o variante, las peculiaridades del ejemplar (entre los impresos) o de la copia, la condición de autógrafo, la autenticidad de la firma (entre los manuscritos), la encuadernación, el ex-libris u otras marcas de propiedad, el origen de los autógrafos o anotaciones marginales... Adquiere verdadero interés en el caso de tratarse de manuscritos o de impresos de difícil identificación, especialmente cuando el manuscrito o el ejemplar posee escasa información sobre él mismo, como sería el caso de muchos incunables, impresos "sine notis", mútilos, o efímeros. Cuando la expertización se realiza sobre una colección de documentos analizando sus valores acumulados como tal colección puede denominarse, en ocasiones, inventario ${ }^{8}$. Éste contiene una sumaria relación ordenada y precisa de las obras examinadas, no incluye valor comercial pero debería contar con la reproducción fotográfica de cada una de las obras para evitar que se pueda mudar cualquiera de los componentes de la colección.

\section{"Cualquier actuación pericial aporta siempre y únicamente un juicio de valor de un profesional sobre un asunto específico"}

b) Tasación: es la actuación pericial que se efectúa para proporcionar el precio de un objeto o de un conjunto de ellos de acuerdo con unos fines y unas características específicas. Aunque incluye muchos datos relativos al documento para avalar el precio establecido por el perito, no proporciona certificación. Es interesante que incluya reproducción de algunas partes de la obra que hayan sido especialmente tratadas en el informe y aquellas que mejor definan la identificación del ejemplar. Una tasación como documento de un experto no puede concretar un valor constante para el objeto en cuestión, por lo que en ella se ha de establecer siempre su validez temporal. Es, por tanto, necesario que se incluya en el documento la validez en el tiempo de la estimación del precio. Por esta causa, en ocasiones se precisa otro tipo de actuación pericial que se denomina constatación. La tasación requiere no sólo el correcto conocimiento del mundo del libro o del documento, sino que exige, además, un análisis de los costes de la circunstancia que produce la tasación y también un buen conocimiento del mercado. Este buen conocimiento del mercado (sin tener en cuenta los dos anteriores factores) ha conducido a algunos interesados en el tema, especialmente libreros, a excluir a los documentalistas de esta actuación, produciéndose una especie de competencia en un mundo que algunos suelen considerar exclusivo. No se tiene en cuenta que esta posición se sitúa en el filo de la ética profesional.

Parece evidente que alguien que está interesado en el negocio, cuando no se es parte de él, no debe emitir su opinión experta sobre el mismo y lo mismo ocurre cuando sus actuaciones pueden influir en su consideración económica de cara al mercado. El documentalista posee los conocimientos básicos para entender sobre los documentos y libros antiguos y raros y los conocimientos suficientes sobre las herramientas para llevar a acabo una estimación acertada del precio de estos documentos o, al menos, estas nociones deberían estar garantizados por su titulación; y, además, no tiene incompatibilidad alguna a la hora de analizar las circunstancias que rodean o generan la tasación. Es cierto que el librero anticuario pone en juego el patrimonio de su empresa en su trabajo, pero no parece imprescindible que la actuación pericial requiera formación en economía, que profesionalmente no les es exigida a los libreros, como tampoco la documental, habida cuenta que el librero, también el anticuario, suele ser un profesional autodidacta.

\section{"Difícilmente puede ser considerado como perito o experto válido el profesional que posteriormente pueda verse beneficiado por su decisión"}

c) Peritación 9 : es la actuación que se centra en el análisis de alguna o de algunas de las características del objeto valorado. Se solicita para averiguar el mérito o el demérito que sufre un libro tras cualquier accidente (inundación, mutilación, vandalismo, incendio...) o actuación realizada sobre él (restauración, reencuadernación...), estableciéndose, además, la apreciación o depreciación del mismo. En consecuencia, se verifica cómo un fenómeno ha afectado a los valores del elemento o unidad de que se trate. Para esto se efectúa una evaluación de los daños o mejoras producidas en el objeto y se establece la variación en el precio del mismo causada por esos daños o mejoras. Suele tratarse de una actuación pericial que sirve para apoyar las pretensiones de las partes de un litigio o para asesorar al juez.

d) Constatación: es la actuación pericial que consiste en el examen de una obra con una relativa relación 
escrita cuando ha habido una tasación anterior sobre la misma. Se trata, más bien, de una actualización de una tasación anterior en cuanto a la cuantificación económica, que se habrá de justificar analizando de manera pormenorizada cualquier factor que haya podido sufrir modificaciones: la moda, la oferta y la demanda, otros parámetros de mercado... Se trata de una actuación que requiere la actualización de los datos aportados en la tasación previa, sobre todo por lo que se refiere a las referencias sobre tasaciones o testigos, utilizados en ella. No precisa reproducciones de la obra, ya que se fundamenta en un dictamen o informe anterior que ya las posee. Si se percibiesen modificaciones en el documento, se trataría de una peritación.

e) Por último, una consulta es una actuación pericial que consiste en una relación verbal sobre la cuestión relativa a la obra u obras con o sin tasación que no se traduce en la confección de un documento escrito. Es el parecer de un experto sobre los valores y precio, o sólo sobre alguno de ellos, de un objeto dado, en este caso de un libro o de un documento. Se trata, de entre las descritas, de la actuación más frecuente y carece, como es lógico, de valor documental, aunque posee el valor informativo que interesa al comitente. Es el primer contacto (y en ocasiones el último) de un experto con un libro.

Si se entra de manera específica en el mundo del libro se puede apreciar mejor la incidencia de las actuaciones periciales como actuación profesional del documentalista.

\section{"La tasación es la actuación pericial que se efectúa para proporcionar el precio de un objeto o de un conjunto de ellos"}

\section{Origen y causa de las actuaciones periciales}

Son numerosas las causas que dan lugar a este tipo de actuaciones. La curiosidad o la necesidad de información (conocer los valores y el precio de sus libros) por parte del propietario a la hora de iniciar un negocio jurídico en el que intervienen esos libros es el principal de los motivos que hace necesaria su expertización o tasación.

El conocimiento de estos factores referidos al libro en su estado actual, el coste de la restauración o de una encuadernación y el precio que puede adquirir tras una restauración o reencuadernación son imprescindibles para la toma de decisiones en el mundo comercial.
La valoración de los ejemplares puede determinar la preferencia de una actuación específica. La información así obtenida puede servir para otras actuaciones posteriores que se realizan sobre libros, en las que estos conocimientos pueden resultar imprescindibles. Se destacan algunos tipos de actuaciones fundamentadas exclusivamente en los negocios jurídicos y actuaciones judiciales y administrativas en las que la determinación pericial de los valores, incluido el valor económico es esencial. De forma muy somera se describen a continuación.

a) El primero de los casos, quizá el más frecuente, se produce cuando el libro es causa de un negocio jurídico de derecho privado. El más destacado y el más habitual es, sin duda, la compraventa. Parten del supuesto de que el vendedor espera minimizar el coste del objeto del negocio jurídico y el comprador maximizarlo. No es preciso que se produzca una tasación profesional ni existe norma que imponga al vendedor supeditarse a una tasación profesional, basta con el acuerdo de comprador y vendedor sin que exista intención de fraude, lo que implica al menos una constatación verbal.

Un segundo caso, por su frecuencia y trascendencia en la transmisión de bienes, es el representado por las subastas. En ellas el obligado a establecer una tasación, en el nivel que sea, es el propietario que acepta la recomendación del profesional de la casa de subastas. Sin embargo, el que determina el precio definitivo es el comprador. Hay que tener en cuenta que tanto el propietario como el comprador son clientes de la casa de subastas que es el proveedor del servicio de mediación. La aparición de la subasta como forma de adquisición supone que el precio establecido por un experto, perito de la casa de subastas, resulta modificado por un, supuestamente, no experto, que es en esta circunstancia el comprador por ser el que realiza la última puja.

El tercero de los casos se produce en los contratos de seguros. En la fase preparatoria la parte que toma el seguro es la que decide el grado de cobertura que desea tener y, de la misma manera, evita posteriores confrontaciones sobre el valor del libro en caso de siniestro. No es el precio del objeto, sino el grado de cobertura lo que decide el asegurado. Debería hablarse del precio de reposición, pero se ha de tener en cuenta que la reposición de un documento antiguo o raro es, en la práctica, imposible. Conviene, por tanto, fijar el precio con la máxima precisión para poder recuperar, en caso de siniestro, no sólo el valor económico del documento o "precio de mercado", sino también el valor económico de los costes imprescindibles para su supuesta reposición, esto es, el "precio de reposición”. En el caso de siniestro pueden plantearse problemas que se habrán de resolver con la intervención de peritos y, en última instancia, ante un juez. 
En cuarto lugar, podría plantearse la necesidad de una tasación profesional cuando se crea una persona jurídica (sociedad) en la que por el motivo que sea intervienen libros. Además, se puede solicitar una tasación en caso de contrato de comodato (préstamo de uso), en el caso de pignoración (prenda), en el caso de pago mediante adjudicación o cesión de bienes y en los negocios "mortis causa", es decir, testamentarías.

\section{"La peritación es la actuación que se centra en el análisis de alguna o algunas de las características del objeto valorado"}

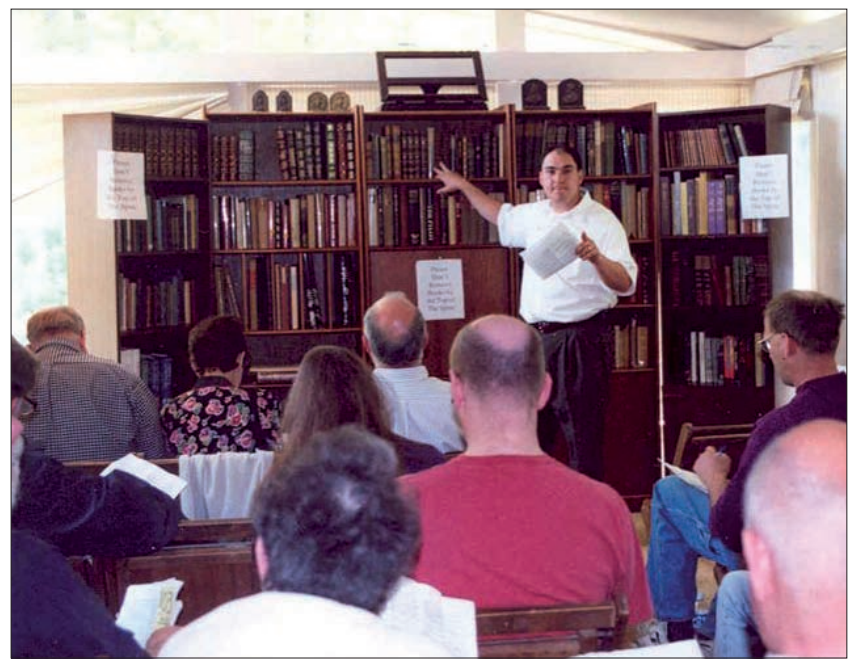

Figura 2. Subasta de libros en Braun and Helmer, Ann Arbor, Michigan

Sin entrar en lo que desde una perspectiva técnica supone la división de una biblioteca, nunca deseable, no parece lo más adecuado dividir una biblioteca heredada estableciendo lotes por el número de volúmenes que la componen sino por el precio de cada uno creando lotes equitativos. La apreciación técnica sobre el interés de un conjunto armonizado según un principio (el que sea), que siempre es mayor que el de los subconjuntos creados, tiene el inconveniente de chocar con la libertad que asiste a un propietario o a un conjunto de ellos para decidir sobre su propiedad.

b) Es necesaria una actuación pericial en el caso de la intervención de la administración como consecuencia de cualquier variable relacionada con el patrimonio, bien sea para la adquisición de bienes patrimoniales (libros, en este caso) por parte de la administración o para la protección del patrimonio, por ejemplo, para autorizar exportaciones.

c) La administración también actúa en procedimientos tributarios en la legislación española. Un caso destacado es el de la comprobación de precios por dación en pago de deudas tributarias (cuando la Agencia Tributaria no se muestra de acuerdo con un valor dado). La Ley prevé que los contribuyentes puedan cancelar sus obligaciones tributarias mediante la donación a las instituciones públicas de libros antiguos, documentos, etc. por lo que es preciso negociar para acordar su tasación. También sería necesaria una tasación para determinar los beneficios fiscales obtenidos por donación de bienes culturales, (es preciso que se contraste con la junta de valoración correspondiente) $)^{10}$.

\section{"La función del experto o perito es una actividad profesional de importancia dentro de las posibles labores de los especialistas en información y documentación"}

d) Es necesaria, también, una actuación pericial cuando se deriva de un mandato judicial o de la solicitud de las partes. Estos mandatos judiciales pueden producirse tanto en el contexto civil para establecer la cuantía económica del procedimiento, como en el penal, en el caso de evaluar los daños causados sobre bienes culturales, en este caso libros. Pero también las partes pueden aportar dictámenes periciales al proceso.

e) Los particulares y las instituciones pueden precisar una evaluación o una certificación sobre un documento con objeto de establecer con claridad qué se posee y cuáles son los valores que caracterizan al documento o al libro antiguo. Esta evaluación o certificación puede verse referida a todo el documento: determinación de la originalidad del ejemplar, o de la elaboración de ejemplares compuestos con otros varios (impuros y bodas)...; o a algunos de los elementos materiales o estructurales del mismo: su encuadernación, anotaciones, ex-libris, ilustraciones, falsificaciones parciales...

f) Algunas de estas actuaciones periciales deben ser aplicadas en los propios centros de información con fines exclusivamente de gestión. El reconocimiento de los valores y del precio de un documento de cara a la toma de decisiones: préstamo para una exposición, seguros, restauración...; o el hecho de obtener el dato elemental del precio de los recursos materiales que posee una institución, entre los que una biblioteca con un importante fondo antiguo no puede ser obviado. Este último dato debe ser contemplado a la hora de gestionar los recursos patrimoniales, lo que repercute de forma destacada en la consideración de la biblioteca como uno de los haberes más importantes de la institución de que se trate. 
g) En ocasiones se impone la curiosidad del propietario que simplemente desea conocer en profundidad el libro o el documento que posee. No se oculta que, detrás de esa curiosidad, suele estar la reserva de datos esenciales de cara a determinadas decisiones por parte del propietario.

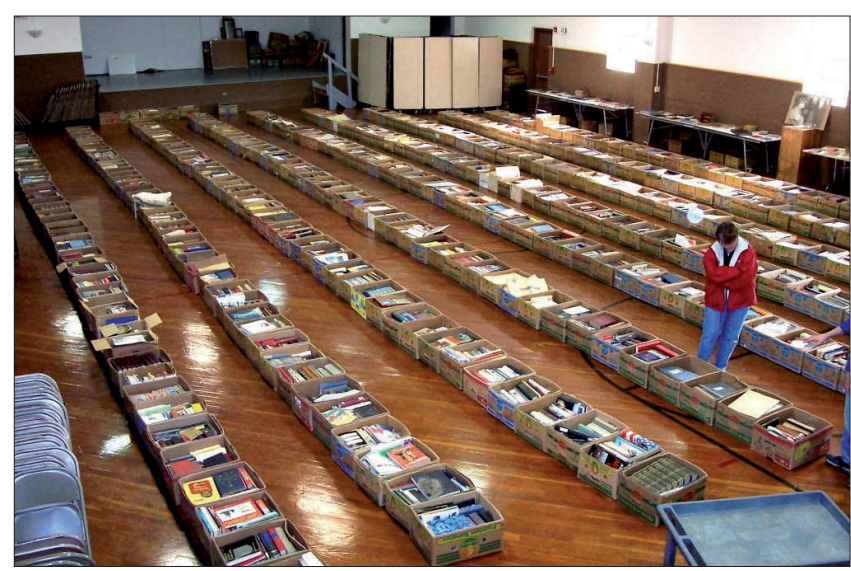

Figura 3. Lotes de libros preparados para subastar por Northeast Auctioneers, Osmond, Nebraska

\section{Método de actuación}

Cualquier actuación de un experto sobre un libro o documento antiguo o raro ha de tener en cuenta un gran número de factores. Esta actuación no es tan elemental como puede parecer y debe conformarse como el fruto de un conjunto de acciones que se llevan a cabo dependiendo del tipo de actuación pericial que se solicita. Se sigue un proceso que puede expresarse en los siguientes pasos, de los que no todos son esenciales en todas las actuaciones:

- Identificar el objeto (el elemento), o mejor, el conjunto al que pertenece el elemento, definiendo sus peculiaridades. Es una de las labores principales y la primera en el proceso. Es el objeto el que esencialmente va a determinar sus valores y su precio.

- Establecer la estimación del valor o valores de una unidad (elemento) de ese conjunto. De esta manera, la valoración del objeto se constituye como uno de los elementos principales del proceso. En este momento intervienen de forma determinante criterios comparativos.

- Identificar los rasgos que hacen que esa unidad sea diferente del resto del conjunto. Se trata de particularidades que, con el transcurso del tiempo y los acontecimientos, singularizan el documento; son casi siempre consecuencia de la esencia del objeto.

- Identificar en el mercado la consideración de las peculiaridades de la unidad documental. Establecer cómo éstas inciden en el precio de otras unidades y en las del libro en cuestión. Sólo se produce en el caso de que sea necesario establecer el precio.

- Determinar el precio definitivo de la unidad y su validez durante un tiempo limitado, que suele ser constante. Sólo se realiza, también, en caso de tasaciones.

- Emitir un informe justificativo de las acciones realizadas que proporcione la información solicitada.

\section{"La constatación consiste en el examen de una obra cuando ha habido una tasación anterior sobre la misma"}

En el mundo de la documentación el proceso de valoración y tasación de los documentos no es automático y se precisan los mismos pasos: identificar la obra y a ser posible la edición, estimar el valor del documento, identificar las peculiaridades del ejemplar, analizar el mercado en función de estas, establecer el precio y emitir un informe o dictamen, bien sea destinado a un uso general o para su uso en un litigio.

\section{Consideraciones finales}

Como puede apreciarse, la función del experto o perito en este tipo de documentos (siendo ésta una condición precisa y necesaria) se conforma como una actividad profesional de importancia dentro de las posibles labores de los especialistas en información y documentación.

Estas labores profesionales se pueden llevar a cabo:

- de forma directa ante clientes particulares en el ejercicio libre de la profesión,

- como trabajador de la institución que precisa de ellas,

- como intermediario ante particulares,

- previa solicitud de las partes o del tribunal en un proceso.

Estas funciones pueden denominarse actuaciones periciales.

\section{Notas}

1. El expolio y sustracción de mapas en la Biblioteca Nacional de España fue la causa del cese fulminante de su directora. Nadie ha sido cesado de la misma manera por una mala gestión con documentos modernos, por perder usuarios o por el hurto de unas decenas de libros anuales.

2. Detrás de unos muros como la Biblioteca de Barcarrota, entre la encuadernación de un libro como las Bulas de Borja o bajo el sudario de una momia como las bulas y el Breviario de Cuéllar. 
3. El comercio del libro en subastas ha sido evaluado en mil millones de dólares durante el año 1999. Guillemet, Pierre-Yves. Les livres précieux: un moyen de diversification des enchères d'art. Memoire de fin d'études, École des Hautes Études Commerciales, Paris, 2001, p. 9.

4. La Ley 1/2000, de 7 de enero, de Enjuiciamiento civil, en su art. 340 permite la actuación como peritos de academias e instituciones culturales y científicas; y lo mismo hace la vigente Ley de enjuiciamiento criminal promulgada por Real decreto de 14 de septiembre de 1882 en su art. 457. Aunque el artículo 458 de la misma norma establece la preferencia de los peritos titulados sobre los que no lo están.

5. En el ámbito procesal el perito debe manifestar, bajo juramento o promesa de decir verdad, que ha actuado y, en su caso, actuará con la mayor objetividad posible, tomando en consideración tanto lo que pueda favorecer como lo que sea susceptible de causar perjuicio a cualquiera de las partes, y que conoce las sanciones penales en las que podría incurrir si incumpliere su deber como perito. Este principio de objetividad es básico en el principio de su actuación.

6. No obstante, la mencionada Ley de enjuiciamiento criminal en su art 459 contempla la imposibilidad de encontrar un segundo perito o la imposibilidad de esperar a la llegada del segundo sin inconvenientes para el curso del sumario.

7. El art. 468 de la Ley de enjuiciamiento criminal considera como causas de recusación: el parentesco de consanguinidad o de afinidad dentro del cuarto grado con el querellante o con el reo, el interés directo o indirecto en la causa o en otra semejante, la amistad íntima o enemistad manifiesta. En cualquier caso, siempre cabe una contra-tasación y siempre habrá de tenerse en cuenta la ética personal.

8. La Academia Española ha definido inventario como: "asiento de los bienes y demás cosas pertenecientes a una persona o comunidad, hecho con orden y precisión". Son estos dos últimos aspectos los que se persiguen en este tipo de actuaciones.

9. Este término puede ser empleado para designar cualquier actuación pericial. La Academia Española lo define como: "trabajo o estudio que hace un perito". Se opta por él ya que es menos usado que el de peritaje con ese mismo fin.

10. Como indica el Real decreto 111/1986, de 10 de enero de desarrollo parcial de la Ley 16/1985, de 25 de junio, del Patrimonio histórico español, Títulos I y IV

\section{Bibliografía}

Ciantelli, Enrico. L'arte e i mercanti: itinerario storico e proposte. Firenze: Cesati, 1986.

Di Cocco, Enzo. La valutazione dei beni economici. Bologna: Calderini, 1960.

Flores-Jimeno, María-del-Rocío. Tratamiento contable del patrimonio cultural. Tesis doctoral. Universidad de Granada, 2005. http://hdl.handle.net/10481/617

Guillemet, Pierre-Yves. Les livres précieux: un moyen de diversification des enchères d'art. Memoire de fin d'études, École des Hautes Études Commerciales: Paris, 2001

Ley de enjuiciamiento criminal, promulgada por Real decreto de 14 de septiembre de 1882 (Gaceta n. 260 de 17 de septiembre de 1882 a Gaceta n. 283 de 10 de octubre de 1882).

http://dgraj.justicia.es/SecretariosJudiciales/docs/lenjuiciamientocriminal. $p d f$

Ley 16/1985, de 25 de junio, del Patrimonio histórico español (BOE n. 155 de 29 de junio de 1985).

http://noticias.juridicas.com/base_datos/Admin/l16-1985.html

Ley 1/2000, de 7 de enero, de Enjuiciamiento civil (BOE n. 7 de 8 de enero de 2000).

http://noticias.juridicas.com/base_datos/Privado/l1-2000.html

Liberati, Stefano. La stampa d'arte: guida al riconoscimento all'attribuzione e alla valutazione. Roma: Palombi, 2005.

Misseri, Salvatore-Corrado. La valutazione delle opere d'arte: metodo e processi. Bologna: Calderini, 1988

Real decreto 111/1986, de 10 de enero, de desarrollo parcial de la Ley 16/1985, de 25 de junio, del Patrimonio histórico español (BOE n. 24 de 28 de enero de 1986).

http://noticias.juridicas.com/base_datos/Admin/rd111-1986.html

Manuel-José Pedraza-Gracia. Universidad de Zaragoza, Facultad de Filosofía y Letras,

C/ Pedro Cerbuna, 12. 50009 Zaragoza.

pedraza@unizar.es

\title{
Próximos temas centrales
}

\author{
Julio 2010 \\ Arquitectura de la información \\ Septiembre 2010 \\ Cooperación de bibliotecas en red \\ Noviembre 2010 \\ Medios de comunicación en internet \\ Enero 2011 \\ Psicología y sociología de la información \\ Marzo 2011 \\ Archivos administrativos e intranets \\ Mayo 2011 \\ Información de las administraciones públicas \\ Julio 2011 \\ Documentación fotográfica \\ Septiembre 2011 \\ Inteligencia competitiva \\ Los interesados pueden remitir notas, artículos, propuestas, publicidad, comentarios, etc. \\ sobre estos temas a: http://recyt.fecyt.es/index.php/EPI/index
}

